GRADIATION\&APPLICATIONS

ISSN 2466-4294 (online) | rad-journal.org

Vol. 2 | Issue $1 \mid$ pp. $20-25$

doi: 10.21175/RadJ.2017.01.005

Original research paper

\title{
COMPARISON OF MEASURED AND CALCULATED OUT-OF-FIELD DOSES IN A PAEDIATRIC ANTHROPOMORPHIC PHANTOM / OUT OF THE BODY SCATTER CONTRIBUTION EVIDENCE*
}

\author{
Firas Ghareeb $^{1^{* *}}$, Sofia Silva ${ }^{1,2}$, Joana Lencart1,2, Fatima Borges ${ }^{2}$, Joao A.M. Santos ${ }^{1,2,3}$ \\ ${ }^{1}$ Instituto Português de Oncologia do Porto Francisco Gentil- EPE Research Center, Porto, Portugal \\ ${ }^{2}$ Medical Physics Department Instituto Português de Oncologia do Porto Francisco Gentil- EPE, Porto, Portugal \\ 3ICBAS, Abel Salazar Biomedical Sciences Institute, University of Porto, Porto, Portugal
}

\begin{abstract}
A pediatric anthropomorphic phantom was irradiated using a standard 3 DCRT treatment plan with and without including the couch in the Treatment Planning System (TPS; Varian Eclipse 13.5, AAA algorithm) calculations. The $2 D$ scatter dose distributions at several planes away from the isocenter were measured using a Gafchromic EBT3 radiochromic film, and compared with the calculated ones at several distances from the isocenter. The out-of-field dose distributions (from TPS and EBT3) differ considerably, particularly where the cranio-caudal beam has left the body but is still near the surface of the phantom and near the couch interception. Further investigation was conducted to estimate the impact of the beam angle on the dose distribution. A $5 \times 5 \mathrm{~cm} 6 \mathrm{MV}$ photon beam was used to irradiate a fiber carbon slab (couch top material) at different angles of incidence and the scattered radiation was measured with an ionization chamber placed $20 \mathrm{~cm}$ away from the isocenter. The results showed a $5 \%$ increase in scattered radiation between the angles of $O^{\circ}$ and $85^{\circ}$. This is an indication that scatter from the couch must be taken into account when estimating out-of-field dose distributions and that the TPS, even when the couch is included in the calculation, is not sufficiently accurate to predict these doses.
\end{abstract}

Key words: Out-of-field dose, couch scatter

\section{INTRODUCTION}

New advances on equipment, methodology and technologies in the field of cancer treatment have increased the number of patient survivors over the past decades. A considerable number of these survivors have been submitted to External Radiotherapy during their treatment course. The extension of the life expectancy following cancer treatment aroused a growing concern on the long term effects of radiation on normal tissues, especially the risk of developing secondary radiation-induced cancer [1-3], in particular, pediatric patients. The life expectancy of a child who have been submitted to External Radiotherapy is increasingly higher [4], and the induction of secondary induced cancer is thus an increased concern. Therefore, a minimization of future risk is being pursued. An estimation of scattered out-of-field dose distribution and the evaluation of the most effective tools is advisable [5]. The out-of-field doses are rather small if compared to in-field doses, but they can cause considerable damage if they were deposited to radiosensitive organs [6-9]. However, Radiotherapy Treatment Planning Systems (TPS) are not commissioned for out-of-field dose calculations and the accuracy of TPS dose calculations is known to decrease beyond the borders of the treatment fields, and the true accuracy of specific TPS for out-of-field dose is not well documented in the literature [10]. Radiochromic film has become an important tool to verify dose distributions in highly conformal radiation therapy such as IMRT $[11,12]$, due to its high sensitivity to a wide range of radiation doses (from $1 \mathrm{cGy}$ to more than $40 \mathrm{~Gy}$ ), its excellent image resolution (5000 dpi), and its ability to produce a two dimensional dose distribution. Because of its characteristics, Gafchromic EBT3 films have been chosen to evaluate out-of-field dose distribution in this study. The main photon contributions to out-of-field doses come from the scattered radiation from the treatment head, which is a function of the treatment head design and collimator scatter radiation, and from the scattered radiation from the interaction of the primary beams with the patient. The later is treatment-dependent and therefore closely influenced by the prescribed dose, number of beams and orientation, correspondent dose distribution inside the patient [13], and for changes in the material which may be in the path of the radiation beam in question such as the radiotherapy couch tops. Couch tops are made to be relatively x-ray translucent, but they still affect the x-ray characteristics even at high energies $[14,15]$. In this study a continuous carbon fiber couch top was used. The carbon fiber used

\footnotetext{
* The paper was presented at the Fourth International Conference on Radiation and Applications in Various Fields of Research (RAD 2016), Niš, Serbia, 2016.

magnox123@gmail.com
} 
in this study was a 29x10xo.2 $\mathrm{cm}$ and it was machined from a Civco Breast Positioning device similar to the material of the couch. This couch top is designed to reduce the delivered dose to patient skin when posterior beams are used. One of the major parameters affecting scatter dose is the amount of material placed in the beams path which increases photon scatter. A detailed comparison beteew the TPS calculated and Gafchromic film measured dose in the out-of-field region was performed and some explanation for the observed difference has been provided.

\section{MATERIALS AND METHODS}

\subsection{Treatment plan}

A treatment plan was designed for a hypothetical brain tumor located in the left side of the brain, using a three 6MV beam configuration, two lateral and one cranio-caudal (see Fig.1). The plan was created on a computed tomography (CT) dataset of the ATOM pediatric five years old anthropomorphic phantom (model 705-c ATOM dosimetry phantom) which is horizontally transected into $26(2.5 \mathrm{~cm}$ thick $)$ slices (see Fig. 2). The plan was generated with Varian Eclipse v.13.5 TPS, using Analytic Anisotropic Algorithm (AAA). Two dose distributions were calculated: one considering the treatment couch and another not considering it.

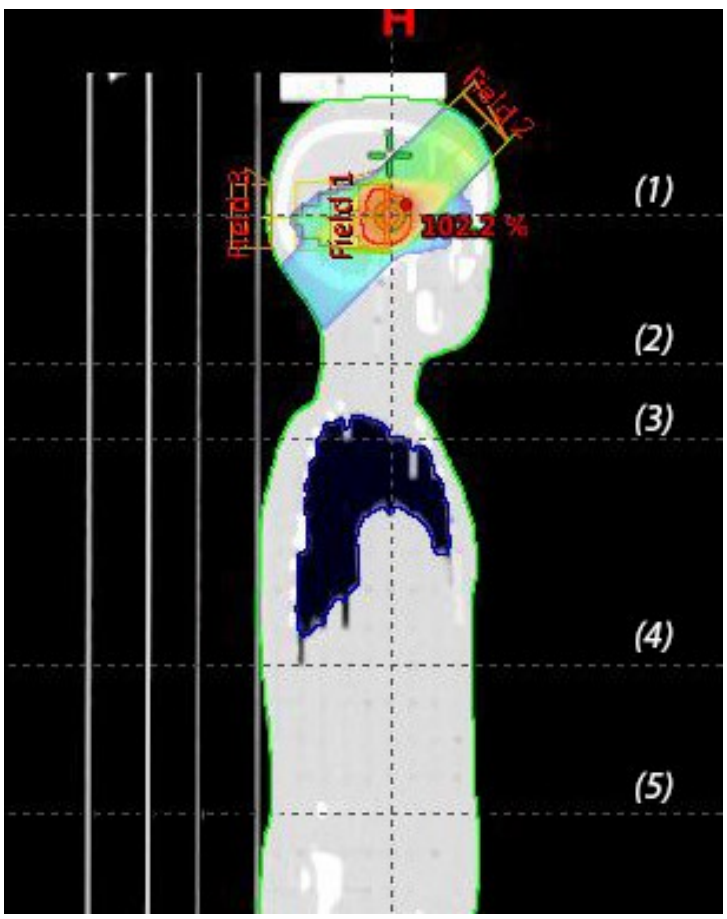

Figure 1. Sagittal view of the treatment plan, showing the cranio-caudal beam orientation relatively to the couch, and the exported planes locations: (1) isocenter level, (2) thyroid

level, (3) lungs level, (4) abdomen level, (5) pelvic level

\subsection{Phantom irradiation}

Gafchromic EBT3 films, were cut to fit the contours of the phantom and placed between contiguous slices of the phantom corresponding to brain, thyroid, lungs, abdomen, and pelvis respectively (see Fig. 2). The phantom was irradiated on a Varian Trilogy (Varian Medical Systems, Palo Alto, California) linear accelerator using the previously described treatment plan. To overcome the lack of film sensitivity at very low doses, the phantom was irradiated with $150 \mathrm{~Gy}$ (10x15 Gy at isocenter).

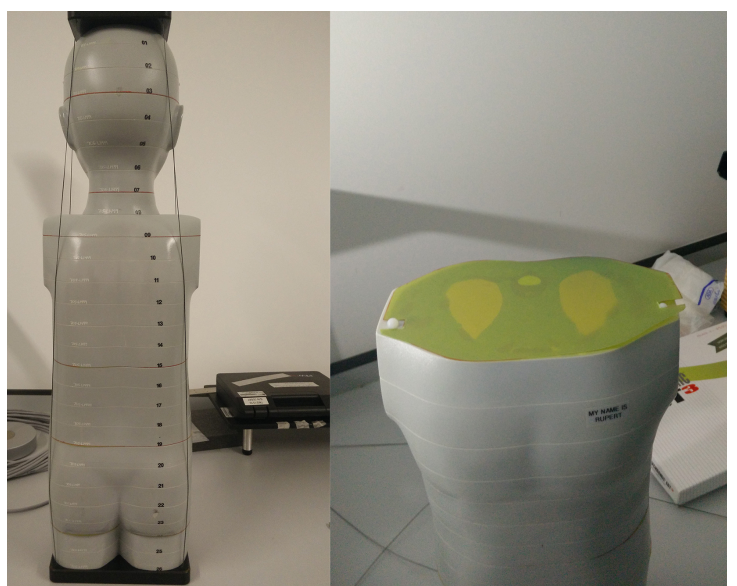

Figure 2. ATOM pediatric five years old anthropomorphic phantom (model 705-c ATOM dosimetry phantom) and EBT3 films placing in between the phantom slices.

\subsection{EBT3 films calibration and analysis}

To calibrate the GAFCHROMIC EBT3 films, films were exposed in a phantom composed of $30 \times 30 \mathrm{~cm}^{2}$ slices of solid water (PTW, Freiburg, Germany) with 5 $\mathrm{cm}$ of the buildup material above and $15 \mathrm{~cm}$ below the film. The source-to-surface distance (SSD) was $100 \mathrm{~cm}$. Film samples were cut $\left(5 \times 5 \mathrm{~cm}^{2}\right)$ and perpendicularly irradiated with $6 \mathrm{MV}(600 \mathrm{MU} / \mathrm{min})$ radiation beam from a Varian DHX-S linac (Varian Medical Systems, Palo Alto, CA). A $10 \times 10 \mathrm{~cm}^{2}$ field size at the isocenter was used. A calibrated ionization chamber PTW Farmer chamber TM30013 (PTW, Freiburg, Germany) was inserted in the phantom $5 \mathrm{~cm}$ below the film plane to check the linac output during the irradiation process and to determine the dose delivered to the film by applying the IAEA-TRS 398 protocol [16]. To obtain a calibration curve, films were exposed at the dose levels from $9.5 \mathrm{cGy}$ to $38.9 \mathrm{~Gy}$.

Films were scanned 24 hours after the irradiation using a flatbed scanner, Epson Expression 1000oXL (Seiko Epson Corp., Nagano, Japan), and its associated software, SILVER FAST $10000 \mathrm{XL}$, was used to read all the films. The scanner was turned on at least 30 minutes before starting the digitations to allow stabilization. Additionally, a sequence of five blank scans was performed in order to warm up the scanner. To minimize the effect of the lateral dependence artifacts (the non-uniform response of the readout due to the light scattering of the scanner lamp caused by particles in the film active layer) black cardboards templates were fitted to the scanner to position films at a central location of the scanner $[17,18]$. Images were acquired in transmission mode and landscape orientation, as recommended by the manufacturer because the lateral response artifact on charge-coupled 
device CCD scanners is smaller in this orientation compared to portrait orientation [17].

The images were converted to units of Optic Density (OD) by DoseLab 6.40 software, using the red and green channels separately. Then the calibration curves between the optical density and the absorbed dose for each channel were created (see Fig 3).

The films which were placed between the anthropomorphic phantom slices were scanned using the same procedure, and the comparison between the measured and calculated dose distributions was performed by DoseLab 6.40 software.

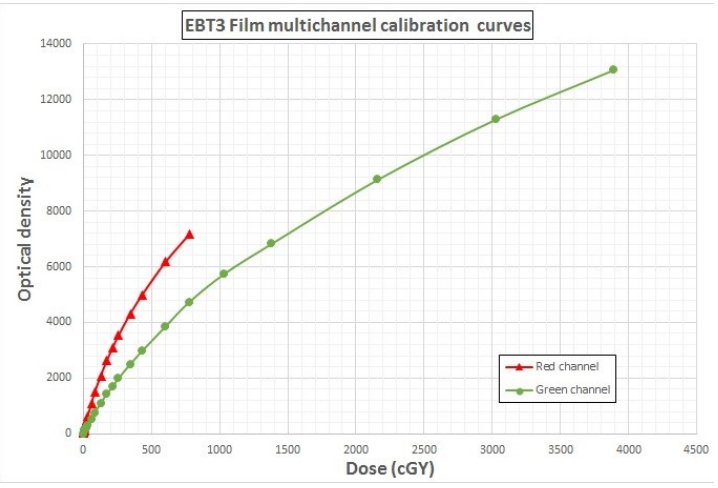

Figure 3. Film calibration curves

Since the red channel is more sensitive for low doses (less than $10 \mathrm{~Gy}$ ), and the green channel is more sensitive for higher doses (more than $10 \mathrm{~Gy}$ ), the green channel calibration curve was used to measure the dose at the isocenter level, where the dose has exceeded the saturation level ( $>40 \mathrm{~Gy}$ ) at some parts of the film, while films in the out-of-field positions have received a small amount of dose (less than $100 \mathrm{cGy}$ for thyroid level, and less than $55 \mathrm{cGy}$ for lungs level ), so the red channel calibration curve is more suitable to evaluate dose at these levels.

\subsection{Out-of-field scattered radiation measurements}

Scatter from carbon fiber material was evaluated in this work as function of incidence angle. To accomplish this task, a $2 \mathrm{~mm}$ thick carbon fiber slab was placed at a fixed distance from the source $(\mathrm{SSD}=100 \mathrm{~cm})$. The plate was tilted and scatter radiation was measured at a fixed distance from the rotation axis in a perpendicular direction (see Fig. 4). For each angle, a fixed number of Monitor Units (200 MU; dose rate of $400 \mathrm{MU} / \mathrm{minute}$ ) with a field size of $5 \times 5 \mathrm{~cm}$, and a beam energy of $6 \mathrm{MV}$, were used using a Varian Trilogy linear accelerator. The dose measurements were acquired using a TM30013 PTW Farmer ionizing chamber with a cylindrical buildup cap made of delrin $\mathrm{CH}_{2} \mathrm{O}$ and has $3.87 \mathrm{~mm}$ of wall thickness, the ionizing chamber and the buildup cap were placed $20 \mathrm{~cm}$ away of the isocenter (see Fig. 4).
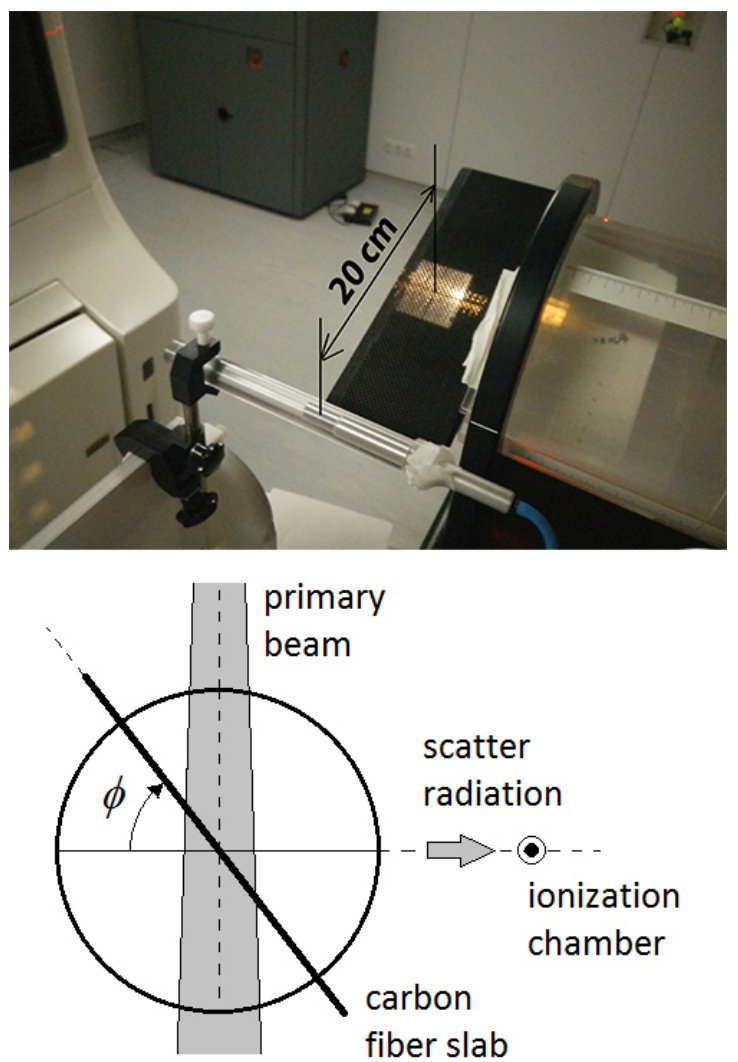

Figure 4. Scattered radiation measurements from a carbon fiber slab

\section{RESULTS AND DISCUSSION}

\subsection{Phantom irradiation}

In Fig. 5, one sees the Gafchromic irradiation analysis at the isocenter level and compared with the planned distribution. Some regions of the film were saturated because the dose exceeded the saturation threshold of EBT3 Gafchromic film (> 40 Gy). On those regions, dose could not be compared. However, out of the saturated region, both dose distributions (TPS and EBT3) matched reasonably well and the transversal antero-posterior profiles were almost identical.

At the thyroid and lung levels $(13.75 \mathrm{~cm}$ and 18.75 $\mathrm{cm}$ respectively from isocenter) the calculated dose distribution without treatment couch and the measured dose distribution differ considerably. 


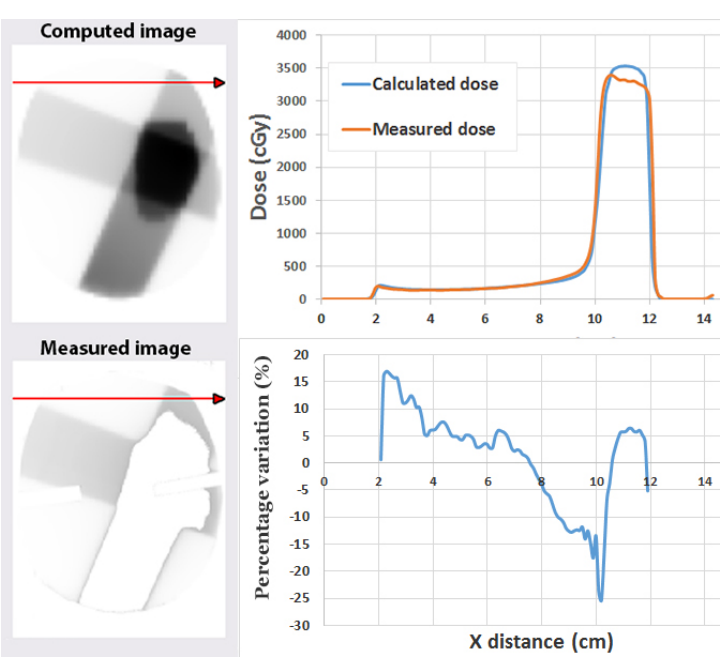

Figure 5. Beam profile at the isocenter level (position 1 in Fig. 1). One applied a 2D gamma function to the surrounding pixels around the profile and $99.8 \%(3 \% / 3 \mathrm{~mm})$ of the pixels (3456 pixels) passed.

The maximum dose in both sets, planned and measured, is located at different positions, resulting sometimes in entirely different dose distributions over the plane. At the thyroid level, a maximum of dose is observed in the posterior region in the film (see Fig. 6.a \& 6.b), while it is located in the left side of the neck in the calculated dose distribution (see Fig. 6.c). Adding an extended calculation volume to the treatment plan, taking into account the couch (see Fig 6.d), increases slightly the left posterior calculated dose showing a very small effect of scattered cranio-caudal beam. However, this effect is largely underestimated by the TPS when compared with the measured distribution.
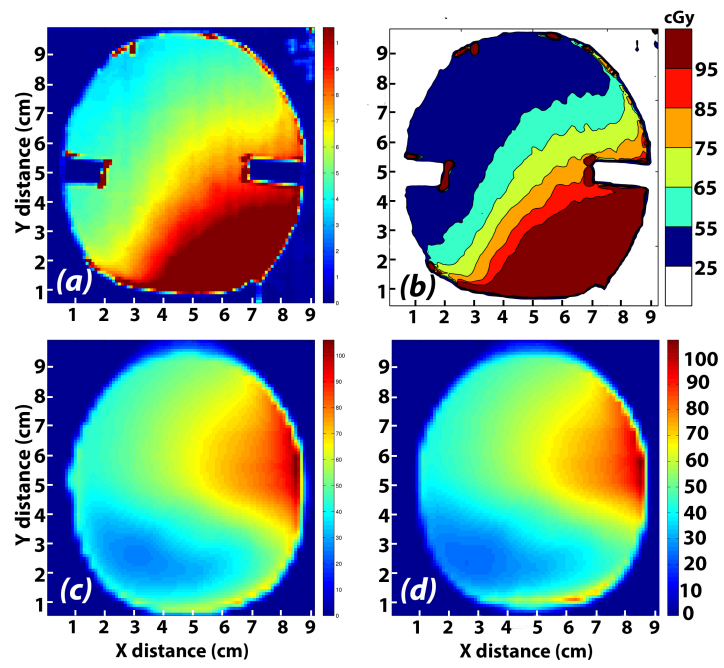

Figure 6. Dose distribution at thyroid level: (a) measured dose distribution, (b) dose contours of measured dose distribution,

(c) calculated dose distribution without including the couch volume, (d) calculated dose distribution with the couch volume included
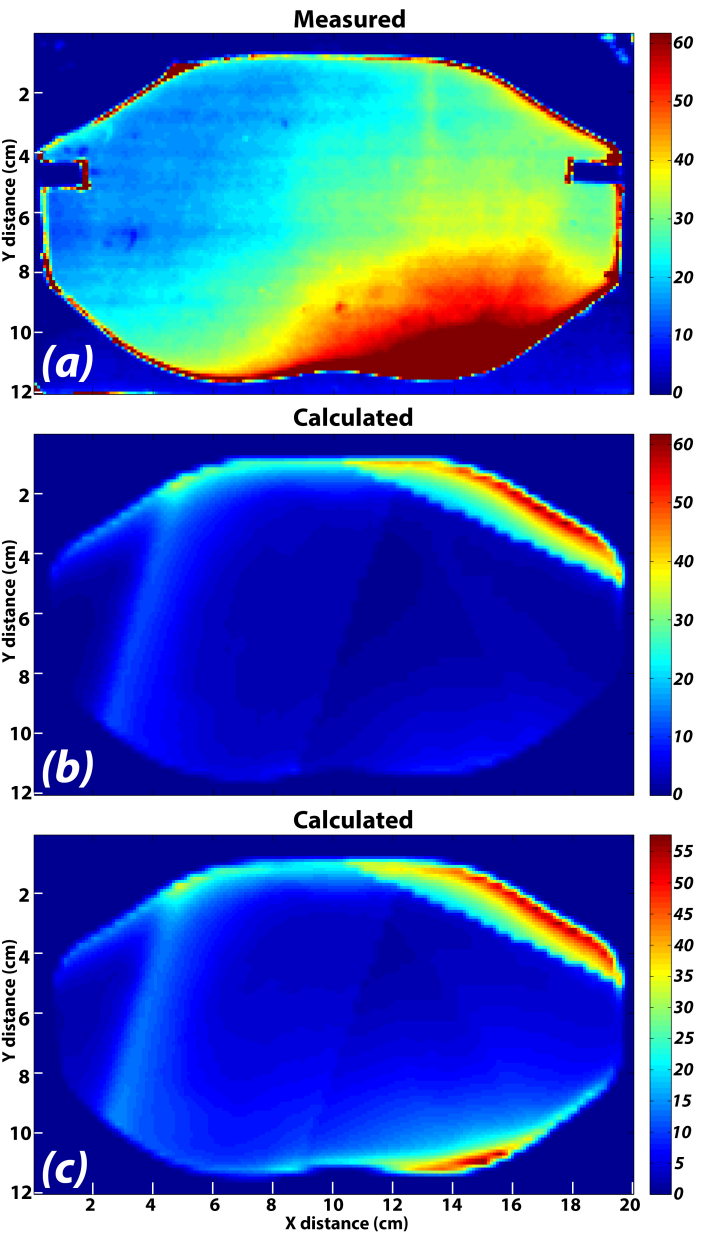

Figure 7. Dose distribution at lungs level: (a) measured dose distribution, (b) calculated dose distribution without including the couch volume, (c) calculated dose distribution with the couch volume included

At the lungs plane, calculated and measured dose distributions are also very different. There is a general trend for the measured dose to be higher than the calculated at these distances (the measured dose is $>50 \%$ higher than the calculated dose at some points, see Fig. 7.a, Fig. 7.b, Fig. 8.a, and Fig. 8.b), particularly where the cranio-caudal beam has left the body but is still near the surface of the phantom.

Furthermore, the calculated dose distribution plan taking into account the couch volume (see Fig. 7.c, and Fig. 8.c) shows to some extent the effect of scattered cranio-caudal beam, but this effect is still underestimated when compared with the measured with EBT3 Gafchromic film.

At abdomen and pelvic level (33.75 \& $43.75 \mathrm{~cm}$ from the isocenter), the dose was too small to be confidently analyzed. 

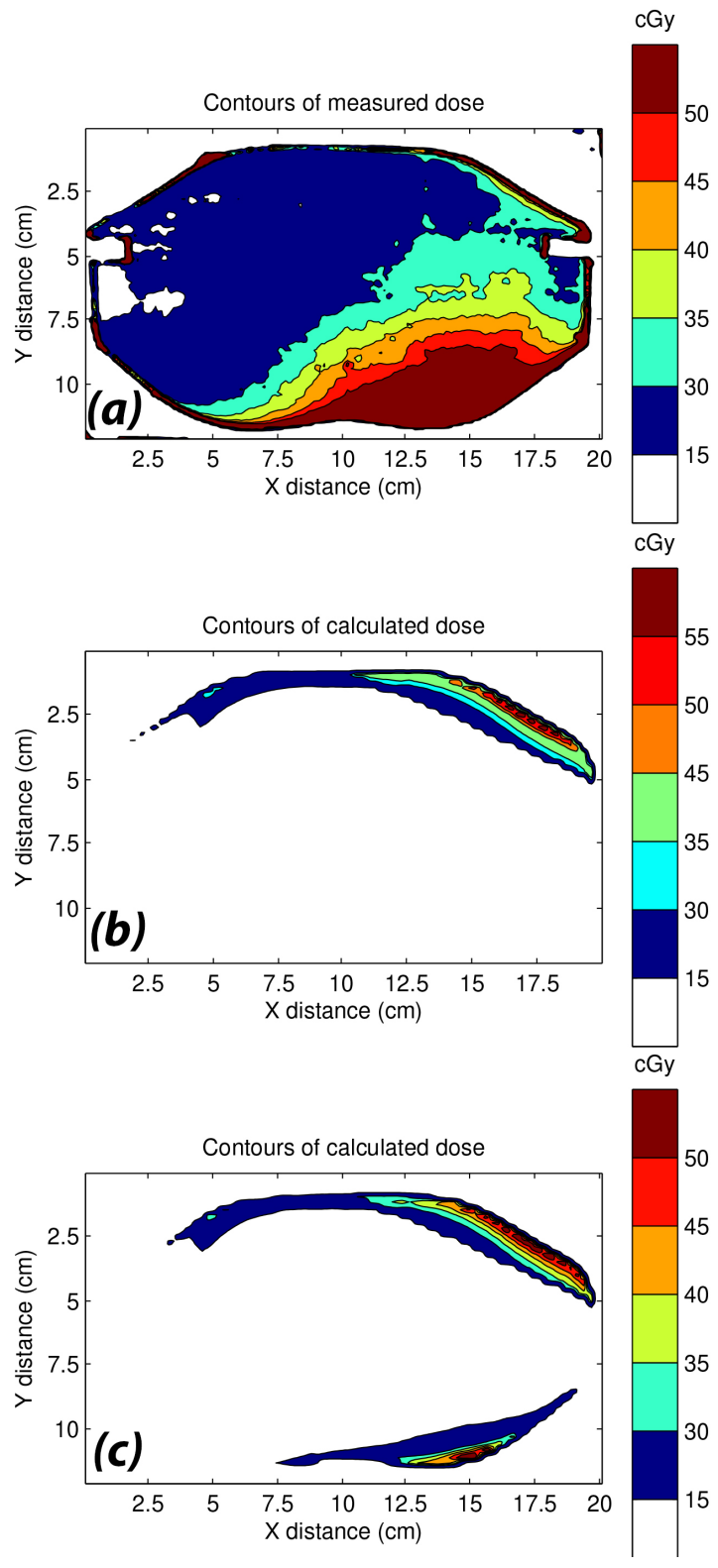

Figure 8. Dose contours at lungs level: (a) measured dose contours, (b) calculated dose contours without including the couch volume, (c) calculated dose contours with the couch volume included

\subsection{Out-of-field scattered radiation measurements}

Since an increase of measured dose was observed on the phantom in proximity of the point where the cranio-caudal beam intercepts the couch, the hypothesis of scatter radiation not being taken into account by the TPS was tested using the methodology described in section 2.4. A first measurement was acquired without the presence of the carbon fiber slab (air). Then it was acquired at different angles of the incidence beam (Fig. 4). The obtained measurements were normalized at the ionization chamber measurement value in the air (without the carbon slab presence), and the results are shown in Fig. 9. The results showed a $5 \%$ increase in scattered radiation when the carbon fiber slab is leveled between $\phi=0^{\circ}$ and $85^{\circ}$ relatively to the horizontal/vertical. Then the scattered radiation started to decrease because the incident field was not entirely contained within the carbon slab anymore (i.e. the incident beam faces a smaller surface to be reflected from, resulting in less scattered radiation). This explains the decrease of the $\phi=90^{\circ}$ point in Fig. 9. One should point that this result applies only to a thin ( $2 \mathrm{~mm})$ carbon fiber plate. In the case of a couch, the thickness and complexity of the entire set can largely increase this effect, and higher doses from scattered radiation should be expected.

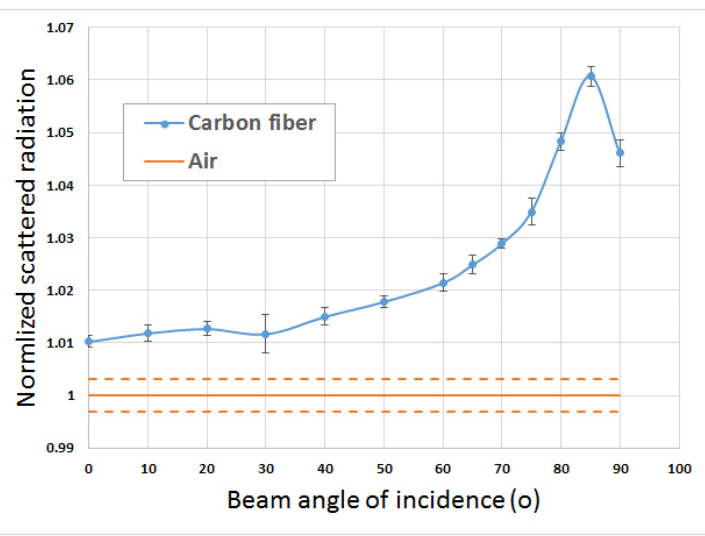

Figure 9. Measurements of scatter radiation

\section{CONCLUSION}

These results show that for secondary radiationinduced cancer risk estimation and out-of-field measurements, the scattered radiation from the couch should be taken into account in dose distribution determination. Even when the treatment couch is considered in the calculation, the Eclipse TPS still shows a trend to largely underestimate the out-of-field dose. Even with a powerful algorithm such as AAA, TPS is still an unreliable tool for out-of-field dose estimation and different tools such as Monte Carlo simulation should be considered for more accurate outof-field dose calculation.

\section{REFERENCES}

1. H. Suit et al., "Secondary carcinogenesis in patients treated with radiation: a review of data on radiationinduced cancers in human, non-human primate, canine and rodent subjects," Radiation research, vol. 167, no. 1, pp. 12-42, 2007.

DOI: $10.1667 /$ RRo527.1 PMid:17214511

2. X. G. Xu, B. Bednarz, and H. Paganetti, "A review of dosimetry studies on external-beam radiation treatment with respect to second cancer induction,", Physics in medicine and biology, vol. 53, no. 13, pp. R193-241, 2008.

DOI: $10.1088 / 0031-9155 / 53 / 13 /$ Ro1

PMid: 18540047

PMCid: PMC4009374

3. W. D. Newhauser, and M. Durante, "Assessing the risk of second malignancies after modern radiotherapy," Nature Reviews Cancer, vol. 11, no. 6, pp. 438-448, 2011. 
F. Ghareeb et al., Comparison of Measured and Calculated Out-of-Field Doses..., Rad. Applic., 2017, 2, 1, 20-25

DOI: $10.1038 /$ nrc3069

PMid: 21593785

PMCid: PMC4101897

4. M. Tubiana, "Can we reduce the incidence of second primary malignancies occurring after radiotherapy? A critical review," Radiotherapy and Oncology, vol. 91, no. 1, pp. 4-15, 2009

DOI: 10.1016/j.radonc.2008.12.016

PMid: 19201045

5. M. L. Taylor, T. Kron, and R. D. Franich, "Assessment of out-of-field doses in radiotherapy of brain lesions in children," International Journal of Radiation Oncology Biology Physics, vol. 79, no. 3, pp. 927-933, 2011.

DOI: $10.1016 /$ j.ijrobp.2010.04.064

PMid: 20732763

6. C. La Tessa et al., "Out-of-field dose studies with an anthropomorphic phantom: Comparison of X-rays and particle therapy treatments," Radiotherapy and Oncology, vol. 105, no. 1, pp. 133-138, 2012.

DOI: 10.1016/j.radonc.2012.04.004 PMid: 22575675

7. M. L. Taylor and T. Kron, "Consideration of the radiation dose delivered away from the treatment field to patients in radiotherapy," Journal of Medical Physics, vol. 36, no. 2, pp. 59-71, 2011.

DOI: $10.4103 / 0971-6203.79686$

PMid: 21731221

PMCid: PMC3119954

8. J. Valentin, "Low-dose extrapolation of radiation-related cancer risk," Annals of the ICRP, vol. 35, no. 4, pp. 1140, 2004.

DOI: 10.1016/j.icrp.2004.12.002

DOI: 10.1016/j.icrp.2005.11.002

PMid: 16782497

9. Health risks from exposure to low levels of ionizing radiation, BEIR VII phase 2, 2006.

Retrieved from:

https://www.nap.edu/catalog/11340/health-risks-fromexposure-to-low-levels-of-ionizing-radiation

Retrieved on: Dec. 12, 2015

10. R. M. Howell et al., "Accuracy of out-of-field dose calculations by a commercial treatment planning system," Physics in medicine and biology, vol. 55 no. 23, p. 6999, 2010.

DOI: $10.1088 / 0031-9155 / 55 / 23 / \mathrm{So} 3$

PMid: 21076191

PMCid: PMC 3152254

11. J. Sorriaux et al., "Evaluation of Gafchromic $₫$ EBT3 films characteristics in therapy photon, electron and proton beams," Physica Medica, vol. 29, no. 6, pp. 599606, 2013.

DOI: 10.1016/j.ejmp.2012.10.001

PMid: 23107430

12. V. C. Borca et al., "Dosimetric characterization and use of GAFCHROMIC EBT3 film for IMRT dose verification," Journal of Applied Clinical Medical Physics, vol. 14, no. 2, 2013.

13. R. Harrison, "Introduction to dosimetry and risk estimation of second cancer induction following radiotherapy," Radiation Measurements, vol. 57, pp. 18, 2013.

DOI: 10.1016/j.radmeas.2013.01.018

14. D. Higgins, P. Whitehurst, and A. Morgan, "The effect of carbon fiber couch inserts on surface dose with beam size variation," Medical Dosimetry, vol. 26, no. 3, pp. 251-254, 2001.

DOI: 10.1016/So958-3947(01)00071-1

15. S. McCormack, J. Diffey, and A. Morgan, "The effect of gantry angle on megavoltage photon beam attenuation by a carbon fiber couch insert," Medical Physics, vol. 32, no. 2, pp. 483-487, 2005.

DOI: $10.1118 / 1.1852792$

PMid: 15789595

16. Absorbed dose determination in external beam radiotherapy: An international code of practice for dosimetry based on standards of absorbed dose to water, Technical Report Series, no. 398, International Atomic Energy Agency, Vienna, 2000.

Retrieved from:

http://wwwpub.iaea.org/MTCD/publications/PDF/TRS398_scr.pdf Retrieved on: Jan. 15, 2016.

17. L. Menegotti, A. Delana, and A. Martignano, "Radiochromic film dosimetry with flatbed scanners: a fast and accurate method for dose calibration and uniformity correction with single film exposure," Medical Physics, vol. 35, no. 7, pp. 3078-3085, 2008. DOI: $10.1118 / 1.2936334$ PMid: 18697531

18. D. Lewis et al., "An efficient protocol for radiochromic film dosimetry combining calibration and measurement in a single scan," Medical Physics, vol. 39, no. 10, pp. 6339-6350, 2012.

DOI: $10.1118 / 1.4754797$

PMid: 23039670 